ISSN 1995-5464 (Print); ISSN 2408-9524 (Online)

https://doi.org/10.16931/1995-5464.202018-17

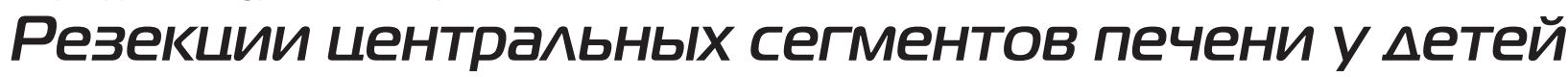

Ахаладзе Д.Г. ${ }^{*}$ *, Качанов Д.Ю. ${ }^{1}$, Ускова Н.Г. ${ }^{1}$, Мелехина О.В. ${ }^{2}$, Меркулов Н.Н. ${ }^{1}$, Талыпов С.P. ${ }^{1}$, Щукин В.В. ${ }^{1}$, Жилкин И.В. ${ }^{1}$, Рабаев Г.С. ${ }^{1}$, Моисеенко Р.А. ${ }^{1}$, Грачев Н.С. ${ }^{1}$

${ }^{1}$ ФГБУ “Национальный медицинский исследовательский центр детской гематологии, онкологии и иммунологии им. Дмитрия Рогачева” Министерства здравоохранения Российской Федерации; 117997, Москва, ул. Саморы Машела, д. 1, Российская Федерация

${ }^{2}$ ГБУЗ “Московский клинический научный центр им. А.С. Логинова” Департамента здравоохранения Москвы; 111123, Москва, шоссе Энтузиастов, д. 86, Российская Федерация

Цель. Анализ ближайших результатов резекций центральных сегментов печени у детей на основании начального опыта.

Материал и методы. Анализировали данные пациентов с различными центрально расположенными новообразованиями печени. В настоящее ретроспективное исследование включены 3 пациента с гепатобластомой, 1 наблюдение мезенхимальной гамартомы печени и 1 пациент с гепатоцеллюлярным раком. За период с марта по октябрь 2018 г. этим пациентам выполнены различные виды центральной резекции печени. Представлены технические аспекты, а также ближайшие результаты лечения.

Результаты. Медиана возраста пациентов составила 78 мес (9 мес - 12 лет). Выполнили сегментэктомию 4b, бисегментэктомию 4b, 5, трисегментэктомию 4-6, мезогепатэктомию (анатомическую сегментэктомию 4, 5, 8) с реконструкцией желчеотведения от задних сегментов печени, мезогепатэктомию с анатомической резекцией S6 и атипичной резекцией S2, 3. Послеоперационные осложнения класса (степени) IIIa по DindoClavien развились у 1 пациента. Сосудистых осложнений и печеночной недостаточности не было. Продолжительность госпитализации варьировала от 9 до 14 дней. Медиана наблюдения составляет 8 мес (7-14 мес). Общая и безрецидивная выживаемость на момент написания работы составляет $100 \%$.

Заключение. Резекция центральных сегментов печени у детей осуществима; операции следует выполнять в центрах, имеющих опыт хирургии печени.

Ключевые слова: печень, опухоли, дети, центральная резекция, мезогепатэктомия.

Ссылка для цитирования: Ахаладзе Д.Г., Качанов Д.Ю., Ускова Н.Г., Мелехина О.В., Меркулов Н.Н., Талыпов С.Р., Щукин В.В., Жилкин И.В., Рабаев Г.С., Моисеенко Р.А., Грачев Н.С. Резекции центральных сегментов печени у детей. Анналы хирургической гепатологии. 2020; 25 (1): 8-17. https://doi.org/10.16931/1995-5464.202018-17.

Авторы заявляют об отсутствии конфликта интересов.

\section{Central segments liver resection for pediatric tumors}

Akhaladze D.G. ${ }^{\text {* }}$, Kachanov D.Y. ${ }^{1}$, Uskova N.G. ${ }^{1}$, Melekhina O.V.', Merkulov N.N. ${ }^{1}$, Talypov S.R. ${ }^{1}$, Schukin V.V. ${ }^{1}$, Zhilkin I.V. ${ }^{1}$, Rabaev G.S. ${ }^{1}$, Moiseenko R.A. ${ }^{1}$, Grachev N.S. ${ }^{1}$

${ }^{1}$ Dmitry Rogachev National Medical Research Center of Pediatric Hematology, Oncology and Immunology Ministry of Health of Russian Federation; 1, Samory Mashela str., Moscow, 117997, Russian Federation

${ }^{2}$ Loginov Moscow Clinical Scientific Center, Department of Health of Moscow; 86, Shosse Entuziastov, 111123, Russian Federation

Aim. To analyze the short-term outcomes of central segments liver resection in children, according to the initial experience.

Methods. The data of patients with different centrally located liver neoplasms were analyzed. This retrospective study included three patients with hepatoblastoma, one observation of mesenchymal hamartoma of the liver and one patient with hepatocellular carcinoma. Different types of central liver resections were carried out in these patients between March and October 2018. The technical aspects and the short-term results are described.

Results. Median age of patients was 78 months (9 months - 12 years). Patients underwent: segmentectomy $4 \mathrm{~b}$, bisegmentectomy $4 \mathrm{~b}, 5$, threesegmentectomy 4, 5, 6, mesohepatectomy (anatomical segmentectomy 4, 5, 8) with biliary reconstruction for posterior liver segments, mesohepatectomy with anatomical segmentectomy 6 and atypical 
S2, 3 resection. Dindo-Clavien grade IIIa postoperative complication developed in one patient. Vascular complications and posthepatectomy liver failure were absent. The hospital stay ranged between 9 to 14 days. The median follow-up period came to 8 months (7-14 months). The overall and event free survival at the time of writing is $100 \%$. Conclusion. Resections of central liver segments in children are feasible and should be carried out in experiences in liver surgery centers.

Keywords: liver, tumors, children, central resection, mesohepatectomy.

For citation: Akhaladze D.G., Kachanov D.Y., Uskova N.G., Melekhina O.V., Merkulov N.N., Talypov S.R., Schukin V.V., Zhilkin I.V., Rabaev G.S., Moiseenko R.A., Grachev N.S. Central segments liver resection for pediatric tumors. Annaly khirurgicheskoy gepatologii = Annals of HPB surgery. 2020; 25 (1): 8-17. (In Russian)

https://doi.org/10.16931/1995-5464.202018-17.

Authors submit that there is no conflict of interest.

\section{Введение}

Анатомическая насыщенность центральных сегментов печени, топография портальных ворот и гепатикокавального конфлюенса объясняют неторопливый темп распространения центральных резекций печени. Необходимо отметить, что в конце XX столетия центральные резекции выполняли спорадически даже у взрослых пациентов. Со временем эти вмешательства набрали популярность, и только в метаанализ, опубликованный в 2017 г. и посвященный сравнению центральных резекций печени с расширенной гемигепатэктомией, были включены 20795 взрослых пациентов, оперированных с 1973 по 2013 г. [1]. Еще медленнее эту операцию внедряют в педиатрическую практику. Одно из первых упоминаний о центральной резекции у детей относится к 1999 г., когда был описан опыт мезогепатэктомии у 3 детей [2]. Авторы заключили, что эти операции воспроизводимы у детей и их следует выполнять в учреждениях, в которых накоплен достаточный опыт хирургии печени у детей. Однако последующий опубликованный опыт центральных резекций у детей ограничивается отдельными описаниями немногочисленных наблюдений.

В эру хирургической гепатологии, когда одной из основных целей операции является сохранение как можно большей массы здоровой паренхимы печени для предупреждения пострезекционной печеночной недостаточности и у ряда пациентов - во избежание трансплантации, попрежнему с большей частотой выполняют расширенную резекцию печени, в том числе у детей [3-6]. Ключевыми факторами, сдерживающими внедрение центральной резекции, остаются необходимость выполнять разделение паренхимы в двух и более плоскостях, бо́льшая кровопотеря и продолжительность операции, а также риск повреждения крупных сосудистых структур и желчных протоков [7-10].

Целью настоящей публикации стало описание выполненных резекций центральных сегментов печени у детей, изложение технических нюансов и представление результатов операций.

\section{Материал и методы}

Ретроспективному анализу подвергнуты 5 пациентов детского возраста, оперированных в ФГБУ “НМИЦ детской гематологии, онкологии и иммунологии им. Дмитрия Рогачева" Минздрава России с марта по октябрь 2018 г. Вмешательства выполнили 3 мальчикам и 2 девочкам. В качестве предоперационного обследования всем пациентам выполняли комплексное УЗИ брюшной полости. Топографию новообразований, их местное распространение, синтопию с сосудистыми структурами и гемодинамику в различные фазы контрастирования оценивали при КТ и МРТ с внутривенным контрастным усилением. При оценке гепатобластомы применяли систему стадирования PRETEXT 2017 [11]. КТ сопровождали оценкой волюметрических характеристик печени, новообразования и будущего остатка печени. Минимальным допустимым объемом будущего остатка печени считали $25 \%$. Функциональный резерв его оценивали при гепатобилиарной сцинтиграфии. Пороговым уровнем функции фрагмента печени считали значение 2,7 \%/мин/ $\mathrm{M}^{2}$. Оба показателя использовали для уточнения необходимости хирургических методов профилактики пострезекционной печеночной недостаточности.

У 2 пациентов при гистологическом исследовании пункционных биоптатов опухоли диагностированы мезенхимальная гамартома печени и гепатоцеллюлярный рак. У 3 пациентов выявлена гепатобластома. Стратификацию в группы риска и лекарственное лечение гепатобластомы осуществляли согласно рекомендациям группы SIOPEL (Société Internationale d'Oncologie Pédiatrique) [12-14]. Двум другим пациентам неоадъювантную полихимиотерапию (нПХТ) не назначали.

Характеристика клинических наблюдений представлена в табл. 1. Пациенту №5, ввиду манифестации болезни механической желтухой, перед нПХТ выполнили чрескожную чреспеченочную холангиостомию модифицированным наружным дренажом под контролем УЗИ и рентгентелевидения. 
Операция. Оперативные вмешательства выполняли в условиях комбинированной анестезии. Стандартным считали J-образный доступ типа Makuuchi, выполненный 4 больным. Пациенту №4 выполнена лапаротомия типа “Мерседес" по Sir R. Calne. После тщательной ревизии брюшной полости и мануальной оценки распространения новообразования в печени выполняли интраоперационное УЗИ. Также уточняли анатомию, топографию печеночных вен, глиссоновых ножек, их синтопию с опухолью. Помимо круглой и серповидной, другие связки печени пересекали лишь при необходимости. Обнажали устья печеночных вен в кавальных воротах. У пациентов №3 и 5 выполняли изолированный обход средней печеночной вены. Выполняли холецистэктомию. Низводили портальную пластинку и приступали к разделению паренхимы у пациентов №1, 2, 4, которым планировали выполнить сегментэктомию 4, бисегментэктомию 4, 5 и трисегментэктомию 4, 5, 6. При планировании резекции анатомическими ориентирами в вертикальной плоскости были портальные фиссуры, а в горизонтальной - плоскость, проведенная через портальные ворота печени. Левая граница резекции проходила в умбиликальной или левой портальной фиссуре и подразумевала пересечение портальных ветвей мелкого диаметра от синуса Rexi к S4. Правой границей, в зависимости от планируемого объема операции, была главная или правая портальная фиссура, которые соответствовали проекциям средней и правой печеночной вен соответственно.

Всем пациентам разделение паренхимы осуществляли чередованием биполярной коагуляции с водоструйной или ультразвуковой деструкцией. Трубчатые структуры, в частности притоки срединной вены от S5 и S8, в плоскости резекции клипировали и пересекали.

Послеоперационный период. Первые 3 сут послеоперационного периода рутинно осуществляли лабораторный контроль и УЗИ с допплерографией. Все осложнения, развившиеся в течение 90 дней после вмешательства, классифицировали по Dindo-Clavien $[15,16]$. К тяжелым относили осложнения III класса (степени) и более. Общую выживаемость рассчитывали со дня хирургического вмешательства до момента смерти, наступившей от любых причин.

\section{Результаты}

Медиана возраста пациентов на момент операции составила 78 мес (9 мес - 12 лет). Медиана объема опухоли до операции составила 136 мл (41-414 мл). Согласно верифицированным диагнозам, у пациентов №2 и 4 хирургическую операцию рассматривали в качестве ключевого этапа лечения. У пациентов №1, 3 и 5 на фоне химиотерапии отмечен ответ опухоли на лече- 
Таблица 2. Сосудистая анатомия печени обследованных пациентов

Table 2. Vascular anatomy of the liver of examined patients

\begin{tabular}{|c|c|c|c|c|c|}
\hline \multirow{2}{*}{$\begin{array}{l}\text { Анатомическая } \\
\text { классификация }\end{array}$} & \multicolumn{5}{|c|}{ № пациента } \\
\hline & 1 & 2 & 3 & 4 & 5 \\
\hline $\begin{array}{l}\text { Артериальная анатомия } \\
\text { по N. Michels }\end{array}$ & Тип 1 & Тип 1 & Тип 1 & Тип 1 & $\begin{array}{c}\text { Тип } 1+ \\
\text { дополнительная } \\
\text { артерия S2,3 от ОПА }\end{array}$ \\
\hline $\begin{array}{l}\text { Кровоснабжение S4 } \\
\text { по G.Varotti }\end{array}$ & LHA type & LHA type & LHA type & RHA type & LHA type \\
\hline $\begin{array}{l}\text { Анатомия воротной вены } \\
\text { по T. Nakamura }\end{array}$ & Тип А & Тип А & Тип А & Тип А & Тип А \\
\hline $\begin{array}{l}\text { Билиарная анатомия } \\
\text { по G. Varotti }\end{array}$ & Тип 1 & Тип 2 & Тип 4a & Тип 1 & Тип 4a \\
\hline
\end{tabular}

Примечание: ОПА - общая печеночная артерия; LHA - left hepatic artery, левая печеночная артерия; RHA right hepatic artery, правая печеночная артерия.

Таблица 3. Предоперационные волюметрические и функциональные показатели будущего остатка печени

Table 3. Preoperative future liver remnant volumetric and functional data

\begin{tabular}{|c|c|c|c|c|}
\hline $\begin{array}{c}\text { № } \\
\text { пациента }\end{array}$ & $\begin{array}{c}\text { Удаляемые } \\
\text { сегменты печени, } \mathrm{S}\end{array}$ & $\begin{array}{c}\text { Остающиеся } \\
\text { сегменты печени, } \mathrm{S}\end{array}$ & $\begin{array}{c}\text { Объем будущего } \\
\text { остатка печени, } \\
\text { см}^{3}(\%)\end{array}$ & $\begin{array}{c}\text { Функциональный } \\
\text { резерв, } \\
\% / \text { мин/м² }\end{array}$ \\
\hline 1 & $4 b$ & $1,2,3,4 a, 5,6,7,8$ & $364(91)$ & $>12$ \\
\hline 2 & $4 b, 5$ & $1,2,3,4 a, 6,7,8$ & $282(83)$ & 12,8 \\
\hline 3 & $4,5,8$ & $1,2,3,6,7$ & $161(55)$ & 3,44 \\
\hline 4 & $4 b, 5,6$ & $1,2,3,4 \mathrm{a}, 7,8$ & $572(76)$ & 8,12 \\
\hline 5 & $4 a, b, 5,8,6$ & 1, большая часть 2,3 и 7 & $430(45)$ & 5,63 \\
\hline
\end{tabular}

ние, который заключался в уменьшении уровня $\alpha$-фетопротеина и сокращении объема опухоли, подтвержденном при УЗИ и КТ, выполненных после II и IV блоков у пациентов, отнесенных к группе стандартного риска (№1, 3), и после блоков А1, А2 (УЗИ) и после блока А3 и (или) блока В (УЗИ, КТ/МРТ) для пациентов группы очень высокого риска (№5) [12]. В среднем объем гепатобластомы на фоне нПХТ сократился на 80\% (68-94\%). Однако ни в одном наблюдении уменьшения стадии PRETEXT (POSTEXT) не отмечено.

Результаты изобразительных методов диагностики позволили установить варианты артериального кровоснабжения печени, воротной вены, строение венозного оттока и билиарного тракта (табл. 2). У всех пациентов источником артериального кровоснабжения печени был чревный ствол. У 4 пациентов наблюдали классический вариант ветвления печеночной артерии $[17,18]$. У пациента №5 был дополнительный источник кровоснабжения левого латерального сектора печени, берущий начало из общей печеночной артерии. Необходимо отметить, что подобного типа артериального кровоснабжения классификации N. Michels, G. Varotti и прочие не предусматривают. У пациента №4 в отличие от других артерия S4 брала начало из правой печеночной артерии.
Bcex пациентов объединял классический вариант ветвления воротной вены - по типу бифуркации (тип А по Т. Nakamura) [19].

Анатомию оттока венозной крови оценивали по классификации S. Orguc [20]. Основное внимание уделяли впадению левой и средней печеночных вен в нижнюю полую вену единым устьем или стволом, строению оттока от правой доли печени, ветвлению притоков срединной вены и топографии фиссуральных вен.

Наиболее вариантной у описываемых пациентов оказалась билиарная анатомия. Два пациента - №1 и 4 - имели типичный вариант формирования общего печеночного и общего желчного протоков. У пациентов №3 и 5 наблюдали низкое впадение протока правого заднего сектора в общий печеночный проток. В наблюдении №2 конфлюенс желчных протоков формировался при слиянии левого печеночного и правых секторальных желчных протоков [21].

В табл. 3 отражены полученные на диагностическом этапе данные КТ-волюметрии и функционального резерва будущего остатка печени. Ни у одного пациента показаний к хирургической профилактике пострезекционной печеночной недостаточности не было.

Пациентам №1, 2 и 4 выполнены представленные в табл. 1 анатомические резекции сегментов печени (рис. 1-3). При определении 


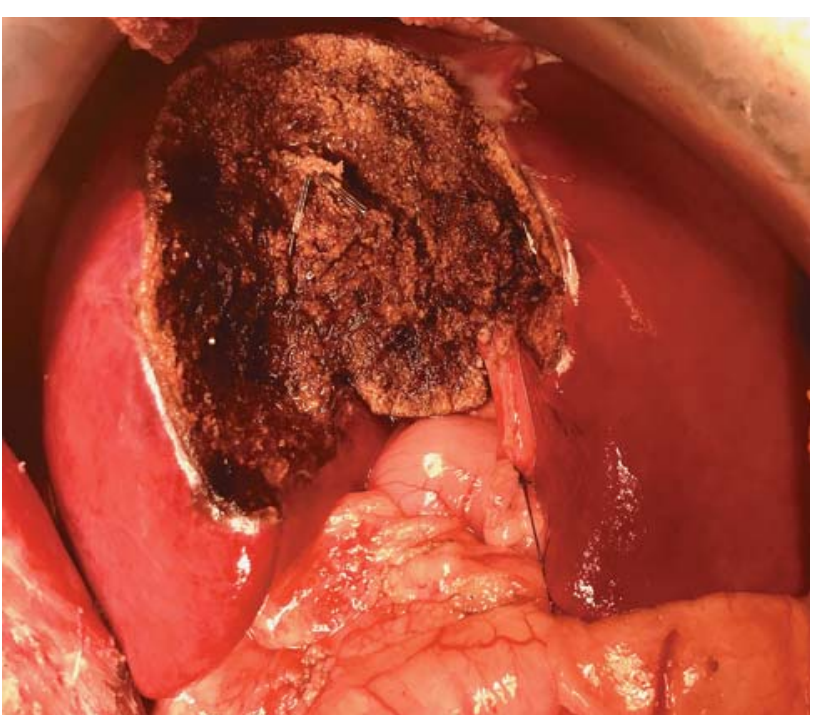

Рис. 1. Интраоперационное фото. Анатомическая сегментэктомия 4b. Вид после завершения операции.

Fig. 1. Intraoperative photo. Anatomical segmentectomy $4 \mathrm{~b}$. The final view of surgery.

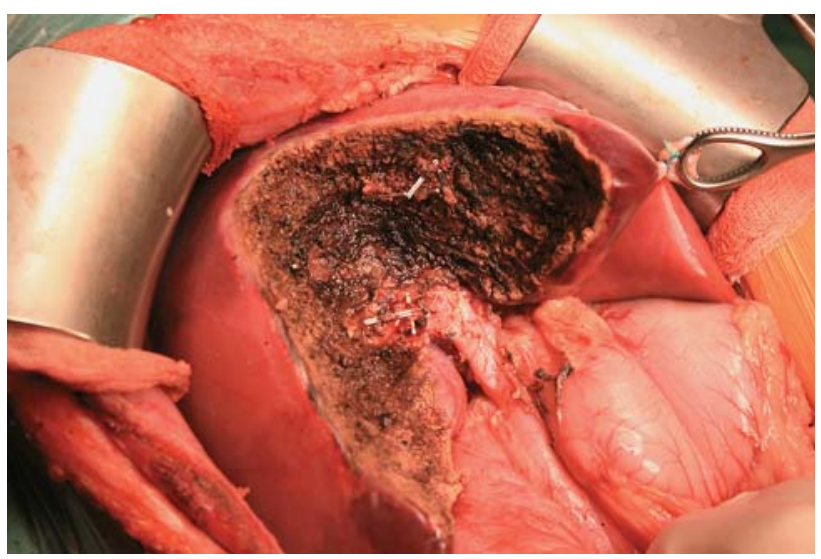

Рис. 2. Интраоперационное фото. Анатомическая бисегментэктомия $4 \mathrm{~b}, 5$. Вид после завершения операции.

Fig. 2. Intraoperative photo. Anatomical bisegmentectomy $4 \mathrm{~b}, 5$. The final view of surgery.

анатомических границ использовали описанные выше ориентиры.

Отдельного внимания заслуживают пациенты №3 и 5, которым выполнены классическая мезогепатэктомия (удаление анатомических сегментов $4,5,8$; рис. 4) и мезогепатэктомия с анатомической резекцией $\mathrm{S} 6$ и атипичной резекцией $\mathrm{S} 2$ и 3 (рис. 5) соответственно. После интраоперационного УЗИ, определения объема предстоящей операции брали в турникет среднюю печеночную вену и после холецистэктомии приступали к диссекции элементов печеночно-двенадцатиперстной связки. Ранее обнажали собственную печеночную артерию с долевыми ветвями и ветвь к S4. Затем выделяли бифуркацию воротной

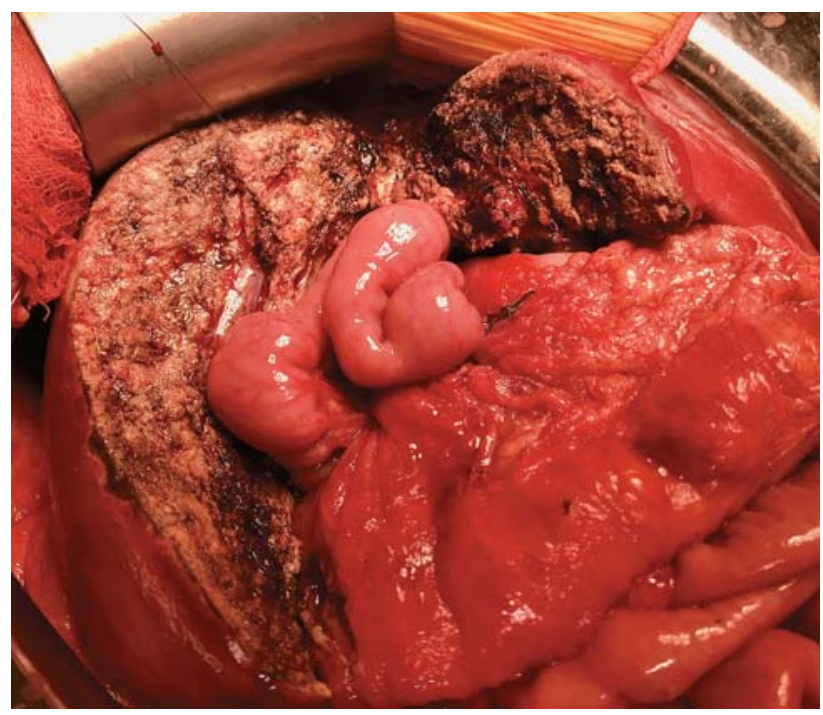

Рис. 3. Интраоперационное фото. Анатомическая трисегментэктомия 4b, 5, 6. Вид после завершения операции.

Fig. 3. Intraoperative photo. Anatomical threesegmentectomy $4 b, 5,6$. The final view of surgery.

вены, секторальные ветви правого заднего и переднего секторов. Последнюю лигировали, пересекали и получали демаркационную линию на границе секторов правой доли печени. Следом осуществляли обработку глиссоновых элементов 4-го сегмента и приступали собственно к резекции печени. У пациента №4 во время резекции выявлена инвазия опухолью протока передних сегментов правой доли. Билиарная анатомия уточнена при интраоперационной холангиографии, а затем выполнена резекция дистальных отделов правых секторальных протоков без нарушения целостности задней стенки конфлюен$\mathrm{ca}$, а также естественного пассажа желчи из левого латерального сектора печени и S1. Реконструкция желчеотведения от задних сегментов печени выполнена путем гепатикоеюностомии на выключенной по Ру петле тощей кишки (см. рис. 4).

Ввиду распространения опухоли на S6 и $\mathrm{S} 2,3$ у пациента №5 для обеспечения радикализма вмешательства левая плоскость резекции проходила по толще кавальной доли печени. Справа выполнена анатомическая сегментэктомия $6 \mathrm{c}$ пересечением сегментарной сосудисто-секреторной ножки и правой печеночной вены на границе S6 и 7. Завершали резекцию пересечением срединной печеночной вены и ушиванием ее культи. У последнего пациента при срочном гистологическом исследовании подтверждено отсутствие атипичных клеток на раневой поверхности 2-го, 3-го, а также 7-го сегмента на его границе с S8 и S6.

Операционные данные. Время операций варьировало от 245 до 775 мин (медиана 445 мин). 


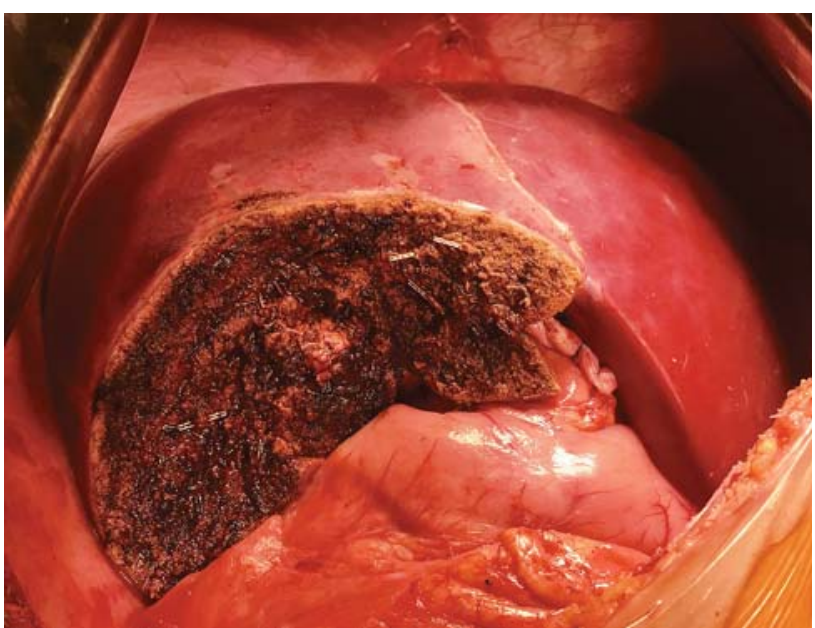

Рис. 4. Интраоперационное фото. Мезогепатэктомия, гепатикоеюностомия. Вид после завершения операции.

Fig. 4. Intraoperative photo. Mesohepatectomy, hepaticojejunostomy. The final view of surgery.

Наиболее продолжительными оказались мезогепатэктомии. Продолжительность сегментэктомии 4, 5, 8, 6 у пациента №5 достигла 775 мин. Продолжительность вмешательства у пациента №3 составила 580 мин ввиду необходимости интраоперационной холангиографии. Гемотрансфузия потребовалась в 3 наблюдениях. Медиана интраоперационной кровопотери составила 100 мл (0-1300 мл). Оперированные пациенты в среднем нуждались в трансфузии 180 мл (0-480 мл) эритроцитарной взвеси.

Во всех наблюдениях готовились выполнять резекцию печени в условиях интермиттирующего пережатия печеночно-двенадцатиперстной связки. Однако прием Прингла применили только у пациента №1, и продолжительность составила 9 мин. Ни одному из пациентов выполнение частичной или тотальной сосудистой изоляции не потребовалось.

Ближайшие результаты. В течение 90 дней послеоперационного периода осложнения разви-

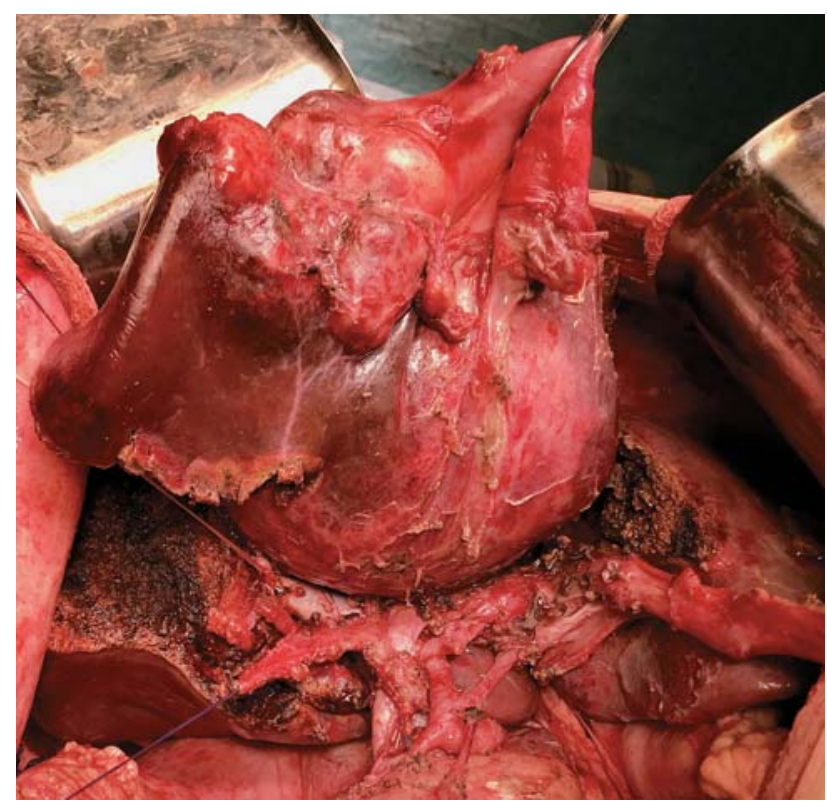

Рис. 5. Интраоперационное фото. Мезогепатэктомия, анатомическая сегментэктомия 6, атипичная резекция $\mathrm{S} 2,3$.

Fig. 5. Intraoperative photo. Mesohepatectomy, anatomical segmentectomy 6 , atypical S2, 3 resection.

лись у 2 включенных в анализ пациентов. Все осложнения были класса (степени) II-IIІа по Dindo-Clavien. У пациента №3 на фоне адъювантной полихимиотерапии развился энтероколит, сопровождавшийся асцитом; осложнение устранено консервативно. У пациента №5 на 3-и сутки послеоперационного периода отмечены признаки PRES-синдрома, а на 28-е сутки после операции он перенес чрескожное дренирование затека желчи под контролем УЗИ. Пострезекционной печеночной недостаточности, сосудистых осложнений, равно как и госпитальной летальности, не было. Медиана продолжительности госпитализации в хирургическом отделении составила 10 дней (9-14 дней). Более подробная информация об осложнениях и данные патоморфологического исследования представлены в табл. 4.

Таблица 4. Характеристика осложнений и результатов патоморфологического исследования Table 4. Postoperative complications and results of a pathomorphological study

\begin{tabular}{|c|c|c|c|c|c|}
\hline \multirow{2}{*}{$\begin{array}{c}\text { № } \\
\text { пациента }\end{array}$} & \multicolumn{2}{|c|}{ Осложнение } & \multirow{2}{*}{ Статус R } & \multirow{2}{*}{$\begin{array}{c}\text { Поражение } \\
\text { лимфатических } \\
\text { узлов }\end{array}$} & \multirow{2}{*}{$\begin{array}{c}\text { Степень } \\
\text { терапевтического } \\
\text { патоморфоза }\end{array}$} \\
\hline & характер & по Dindo-Clavien & & & \\
\hline 1 & - & 0 & 0 & нет & 4 \\
\hline 2 & - & 0 & 0 & нет & - \\
\hline 3 & $\begin{array}{c}\text { Энтероколит, } \\
\text { асцит }\end{array}$ & II & 0 & нет & 2 \\
\hline 4 & - & - & 0 & нет & - \\
\hline 5 & $\begin{array}{l}\text { PRES-синдром, } \\
\text { скопление желчи }\end{array}$ & II/IIIa & 0 & нет & 3 \\
\hline
\end{tabular}


Медиана наблюдения за пациентами составляет 8 мес (7-14 мес). Общая и безрецидивная выживаемость составляет 100\%. Другие показатели выживаемости оценивать преждевременно, поскольку к моменту подготовки статьи только пациентка №1 пережила 1 год.

\section{Обсуждение}

Практически во всех работах, в которых рассмотрен тот или иной объем резекции центральных сегментов, такие вмешательства характеризуют как наиболее сложные в хирургии печени или даже опасные [22]. Эти работы объединяет и аргументация сложности операций. Все авторы сходятся во мнении, что в отличие от многих других центральные резекции печени сложны ввиду близости кавальных и портальных ворот, тесной синтопии с печеночными венами, наличия 2 и более плоскостей резекции, что может провоцировать формирование желчных затеков и билиарных свищей, равно как и ранение сегментарных и (или) секторальных протоков. Ограничивают показания к центральным резекциям и малый отступ от края опухоли, повышенная кровоточивость раневых поверхностей печени, бо́льший объем кровопотери, бо́льшая потребностьвгемотрансфузииипродолжительность госпитализации [2, 6, 23-29]. Вероятно, эти обстоятельства являются причиной отказа от центральных резекций в пользу расширенной гемигепатэктомии, чем можно объяснить ограниченное число публикаций, посвященных этим операциям у детей $[2,6,22,29]$.

В то же время все авторы помимо недостатков выделяют схожие преимущества центральных резекций, основным из которых является сохранение достаточного объема паренхимы органа. Анализ опыта в детской популяции пациентов показывает, что волюметрические характеристики опубликовали только Y. Ohnо и соавт. [22]. После резекции 4-го, 8-го и частично 5-го сегментов печени по поводу гепатобластомы у ребенка 11 мес сохранено 87\% паренхимы вместо $44 \%$, если бы предпочтение было отдано расширенной правосторонней гемигепатэктомии. Необходимо отметить, что и у взрослых пациентов, у которых опыт центральных резекций существенно больше, основным преимуществом центральной резекции является сохранение объема паренхимы печени. Это особенно актуально для предупреждения пострезекционной печеночной недостаточности и сохраняет перспективы при необходимости ререзекции печени [23, $30,31]$. С этой точки зрения интерес представляют данные КТ-волюметрии и функциональный резерв будущего остатка печени у анализируемых пациентов. Как видно из табл. 3, угрозы "small-for-size syndrome", а следовательно, печеночной недостаточности не было даже у пациен- та №5, перенесшего максимальный из представленных объем резекции печени. При пороговом значении функционального резерва в 2,7 \%/ мин/м ${ }^{2}$ пациент продемонстрировал удовлетворительный результат даже после нПХТ по протоколу SIOPEL-4 для пациентов группы очень высокого риска и после механической желтухи, предшествовавшей терапии.

Цифры, демонстрируемые табл. 3, свидетельствуют о том, что волюметрические характеристики, а главное - функция будущего остатка печени (исследуемая впервые в детской популяции) позволили бы перенести пациентам даже расширенную гемигепатэктомию без риска развития печеночной недостаточности. Однако исчерпывающие данные изобразительных методов диагностики, в том числе представление об анатомии афферентных и эфферентных сосудов печени, и в равной степени понимание строения оттока желчи вкупе с бережной техникой диссекции вне- и внутрипеченочных трубчатых структур позволили избежать серьезных осложнений. Любые спорные аспекты билиарной анатомии следует рассматривать при интраоперационной холангиографии. У пациентов №3 и 5 она увеличила продолжительность вмешательства, но позволила принять своевременное решение о формировании билиодигестивного соустья у пациента №3 и воздержаться от такового у пациента №5.

\section{Заключение}

Необходимо отметить, что различные варианты резекций центральных сегментов печени возможны у детей любого возраста и должны выполняться в центрах экспертного уровня. Этот тезис подтверждают продемонстрированные ближайшие результаты подобных операций. Для формулировки окончательных выводов о резекциях центральных сегментов необходим анализ большего числа наблюдений, сравнение результатов с расширенными резекциями печени, аналогично исследованиям у взрослых больных [31], а также оценка отдаленных результатов.

\section{Участие авторов}

Ахаладзе Д.Г. - разработка дизайна статьи, написание текста, редактирование, ответственность за целостность всех частей работы.

Качанов Д.Ю. - разработка дизайна статьи, редактирование текста.

Ускова Н.С. - сбор данных, редактирование текста статьи.

Мелехина О.В. - редактирование текста.

Меркулов Н.Н. - сбор данных, составление таблиц.

Талыпов С.Р. - редактирование текста, разработка концепции статьи.

Щукин В.В. - обработка численных переменных. 
Жилкин В.В. - написание текста статьи, формирование списка используемой литературы.

Рабаев Г.С. - сбор и обработка материала.

Моисеенко Р.А. - сбор данных пациентов.

Грачев Н.С. - дизайн статьи, утверждение окончательного варианта статьи.

\section{Authors participation}

Akhaladze D.G. - concept and design of the study, writing text, editing, responsibility for the integrity of all parts of the article.

Kachanov D.Y. - concept and design of the study, editing.

Uskova N.G. - data collection, editing.

Melekhina O.V. - editing.

Merkulov N.N. - data collection, tabulation.

Talypov S.R. - editing, concept of the study.

Schukin V.V. - processing of the variables.

Zhilkin I.V. - writing text, collection of the references.

Rabaev G.S. - data collection and processing.

Moiseenko R.A. - patient's data collection.

Grachev N.S. - design of the study, approval of the final version of the article.

\section{Список литературы [References]}

1. Chan J., Perini M., Fink M., Nikfarjam M. The outcomes of central hepatectomy versus extended hepatectomy: a systematic review and meta-analysis. HPB (Oxford). 2018; 20 (6): 487-496. https://doi.org/10.1016/j.hpb.2017.12.008.

2. La Quaglia M.P., Shorter N.A., Blumgart L.H. Central hepatic resection for pediatric tumors. J. Pediatr. Surg. 2002; 37 (7): 986-989.

3. Fischer L., Cardenas C., Thorn M., Benner A., Grenacher L., Vetter M., Lehnert T., Klar E., Meinzer H.P., Lamadé W. Limits of Couinaud's liver segment classification: a quantitative computer-based three-dimensional analysis. J. Comput. Assist. Tomogr. 2002; 26 (6): 962-967.

4. Iwatsuki S., Starzl T.E. Personal experience with 411 hepatic resections. Ann. Surg. 1988; 208 (4): 421-434.

5. Fong Y., Fortner J., Sun R.L., Brennan M.F., Blumgart L.H. Clinical score for predicting recurrence after hepatic resection for metastatic colorectal cancer: analysis of 1001 consecutive cases. Ann. Surg. 1999; 230 (3): 309-318.

6. Amesty M.V., Chocarro G., Vilanova Sánchez A., Nuñez Cerezo V., de la Torre C.A., Encinas J.L., Gamez Arance M., Hernández F., Lopez Santamaria M. Mesohepatectomy for centrally located tumors in children. Eur. J. Pediatr. Surg. 2016; 26 (1): 128-132. https://doi.org/10.1055/s-0035-1568995.

7. Wu C.C., Ho W.L., Chen J.T., Tang C.S., Yeh D.C., Liu T.J., Peng F.K. Mesohepatectomy for centrally located hepatocellular carcinoma: an appraisal of a rare procedure. J. Am. Coll. Surg. 1999; 188 (5): 508-515.

8. Chen X.P., Qiu F.Z., Lau W.Y., Zhang B.X., Chen Y.F., Zhang W.G., He S.Q. Mesohepatectomy for hepatocellular carcinoma: a study of 256 patients. Int. J. Colorectal. Dis. 2008; 23 (5): 543-546.

9. Cheng C.H., Yu M.C., Wu T.H., Lee C.F., Chan K.M., Chou H.S., Lee W.C. Surgical resection of centrally located large hepatocellular carcinoma. Chang Gung Med. J. 2012; 35 (2): 178-191.
10. Sotiropoulos G.C., Lang H., Molmenti E.P., Kaiser G.M., Paul A., Broelsch C.E. Partial or complete mesohepatectomy combined with resection of the hilar bifurcation in cases of Klatskin tumors: a reasonable strategy? Am. J. Surg. 2009; 198 (2): 297-298. https://doi.org/10.1016/j.amjsurg.2008.11.022.

11. Towbin A.J., Meyers R.L., Woodley H., Miyazaki O., Weldon C.B., Morland B., Hiyama E., Czauderna P., Roebuck D.J., Tiao G.M. 2017 PRETEXT: radiologic staging system for primary hepatic malignancies of childhood revised for the Paediatric Hepatic International Tumour Trial (PHITT). Pediatr. Radiol. 2018; 48 (4): 536-554. https://doi.org/10.1007/s00247-018-4078-z.

12. Aronson D.C., Czauderna P., Maibach R., Perilongo G., Morland B. The treatment of hepatoblastoma: Its evolution and the current status as per the SIOPEL trials. J. Indian Assoc. Pediatr. Surg. 2014; 19 (4): 201-207. https://doi.org/10.4103/0971-9261.142001.

13. Zsiros J., Brugieres L., Brock P., Roebuck D., Maibach R., Zimmermann A., Childs M., Pariente D., Laithier V., Otte J.B., Branchereau S., Aronson D., Rangaswami A., Ronghe M., Casanova M., Sullivan M., Morland B., Czauderna P., Perilongo G. International Childhood Liver Tumours Strategy Group (SIOPEL). Dose-dense cisplatin-based chemotherapy and surgery for children with high risk hepatoblastoma (SIOPEL-4): a prospective, single-arm, feasibility study. Lancet Oncol. 2013; 14 (9): 834-842. https://doi.org/10.1016/S1470-2045(13)70272-9.

14. Liu A.P.Y., Ip J.J.K., Leung A.W.K., Luk C.W., Li C.H., Ho K.K.H., Lo R., Chan E.K.W., Chan A.C.Y., Chung P.H.Y., Chiang A.K.S. Treatment outcome and pattern of failure in hepatoblastoma treated with a consensus protocol in Hong Kong. Pediatr. Blood Cancer. 2019; 66 (1): e27482. https://doi.org/10.1002/pbc.27482.

15. Dindo D., Demartines N., Clavien P.A. Classification of surgical complications: a new proposal with evaluation in a cohort of 6,336 patients and results of a survey. Ann. Surg. 2004; 240 (2): 205-213.

16. Clavien P.A., Barkun J., de Oliveira M.L., Vauthey J.N., Dindo D., Schulick R.D., de Santibanes E., Pekolj J., Slankamenac K., Bassi C., Graf R., Vonlanthen R., Padbury R., Cameron J.L., Makuuchi M. The Clavien-Dindo classification of surgical complications: five-year experience. Ann. Surg. 2009; 250 (2): 187-196. https://doi.org/10.1097/SLA.0b013e3181b13ca2.

17. Michels N.A. Observations on the blood supply of the liver and gallbladder (200 dissections). In: Blood supply and anatomy of the upper abdominal organs, with a descriptive atlas. Philadelphia, PA: Lippincott, Williams \& Wilkins. 1955; 547, 39 p.

18. Michels N.A. Newer anatomy of the liver and variant blood supply and collateral circulation. Am. J. Surg. 1966; 112 (3): 337-347. https://doi.org/10.1016/0002-9610(66)90201-7.

19. Nakamura T., Tanaka K., Kiuchi T., Kasahara M., Oike F., Ueda M., Kaihara S., Egawa H., Ozden I., Kobayashi N., Uemoto S. Anatomical variations and surgical strategies in right lobe living donor liver transplantation: lessons from 120 cases. Transplantation. 2002; 73 (12): 1896-1903.

20. Orguc S., Tercan M., Bozoklar A., Akyildiz M., Gurgan U., Celebi A., Nart D., Karasu Z., Icoz G., Zeytunlu M., Yuzer Y., Tokat Y., Kilic M. Variations of hepatic veins: helical computerized tomography experience in 100 consecutive living liver donors with emphasis on right lobe. Transplant. Proc. 2004; 36 (9): 2727-2732. https://doi.org/10.1016/j.transproceed.2004.10.006. 
21. Varotti G., Gondolesi G.E., Goldman J., Wayne M., Florman S.S., Schwartz M.E., Miller C.M., Sukru E. Anatomic variations in right liver living donors. J. Am. Coll. Surg. 2004; 198 (4): 577-582.

22. Ohno Y., Furui J., Kanematsu T. Is a modified central bisegmentectomy a volume-saving operation for pediatric hepatoblastoma? J. Pediatr. Surg. 2004; 39 (1): E13-16.

23. Lee S.Y. Central hepatectomy for centrally located malignant liver tumors: A systematic review. World J. Hepatol. 2014; 6 (5): 347-357. https://doi.org/10.4254/wjh.v6.i5.347.

24. Czauderna P., Otte J.B., Aronson D.C., Gauthier F., Mackinlay G., Roebuck D., Plaschkes J., Perilongo G. Guidelines for surgical treatment of hepatoblastoma in the modern era recommendations from the Childhood Liver Tumour Strategy Group of the International Society of Pediatric Oncology (SIOPEL). Eur. J. Cancer. 2005; 41 (7): 1031-1036. https://doi.org/10.1016/j.ejca.2005.02.004.

25. Le N., Rivard D.C., Rentea R.M., Manalang M., Andrews W., Kane B., Hendrickson R.J. Right trisegmentectomy after portal vein embolization in a high-risk toddler with hepatoblastoma. Pediatr. Surg. Int. 2018; 34 (5): 573-578. https://doi.org/10.1007/s00383-018-4250-4.

26. Guérin F., Gauthier F., Martelli H., Fabre M., Baujard C., Franchi S., Branchereau S. Outcome of central hepatectomy for hepatoblastomas. J. Pediatr. Surg. 2010; 45 (3): 555-563. https://doi.org/10.1016/j.jpedsurg.2009.09.025.

27. Li J., Wang C., Song J., Chen N., Jiang L., Yang J., Yan L. Mesohepatectomy versus extended hemihepatectomies for centrally located liver tumors: a meta-analysis. Sci. Rep. 2017; 7 (1): 9329. https://doi.org/10.1038/s41598-017-09535-0.

28. Chen X., Li B., He W., Wei Y.G., Du Z.G., Jiang L. Mesohepatectomy versus extended hemihepatectomy for centrally located hepatocellular carcinoma. Hepatobiliary Pancreat. Dis. Int. 2014; 13 (3): 264-270. https://doi.org/10.1016/S1499-3872(14)60253-8.

29. Chowdappa R., Sagar R.C., Ramesh S., Appaji L., Maneya P., Mukundapai M. Central hepatectomy (mesohepatectomy) by Double Liver Hanging Maneuver (DLHM) in a child with hepatoblastoma. Indian J. Surg. Oncol. 2019; 10 (1): 46-49. https://doi.org/10.1007/s13193-018-0823-X.

30. Hu R.H., Lee P.H., Chang Y.C., Ho M.C., Yu S.C. Treatment of centrally located hepatocellular carcinoma with central hepatectomy. Surgery. 2003; 133 (3): 251-256.

31. Chouillard E., Cherqui D., Tayar C., Brunetti F., Fagniez P.L. Anatomical bi- and trisegmentectomies as alternatives to extensive liver resections. Ann. Surg. 2003; 238 (1): 29-34.

\section{Сведения об авторах [Authors info]}

Ахаладзе Дмитрий Гурамович - канд. мед. наук, руководитель группы торакоабдоминальной хирургии ФГБУ “НМИЦ детской гематологии, онкологии и иммунологии им. Дмитрия Рогачева” Минздрава России. https://orcid.org/0000-0002-1387-209X. E-mail: d.g.akhaladze@gmail.com.

Качанов Денис Юрьевич - доктор мед. наук, заведующий отделением клинической онкологии ФГБУ “НМИЦ детской гематологии, онкологии и иммунологии им. Дмитрия Рогачева” Минздрава России.

https://orcid.org/0000-0002-3704-8783. E-mail: kachanov78@gmail.com.

Ускова Наталья Геннадьевна - канд. мед. наук, врач детский хирург отделения онкологии и детской хирургии ФГБУ “НМИЦ детской гематологии, онкологии и иммунологии им. Дмитрия Рогачева” Минздрава России.

https://orcid.org/0000-0001-9424-1646. E-mail: nataliyauskova@gmail.com.

Мелехина Ольга Вячеславовна - канд. мед. наук, врач-хирург отделения рентгенхирургических методов диагностики и лечения ГБУЗ “Московский клинический научный центр им. А.С. Логинова” Департамента здравоохранения Москвы. https://orcid.org/0000-0002-3280-8667. E-mail: o.melekhina@mknc.ru

Меркулов Николай Николаевич - врач детский хирург отделения онкологии и детской хирургии ФГБУ “НМИЦ детской гематологии, онкологии и иммунологии им. Дмитрия Рогачева” Минздрава России.

https://orcid.org/0000-0003-0404-6420. E-mail: dr.mernich@yandex.ru

Талыпов Сергей Римович - канд. мед. наук, врач детский хирург отделения онкологии и детской хирургии ФГБУ “НМИЦ детской гематологии, онкологии и иммунологии им. Дмитрия Рогачева” Минздрава России.

http://orcid.org/0000-0002-5308-6544. E-mail: riumych@yandex.ru

Щукин Владислав Владимирович - канд. мед. наук, заведующий отделением анестезиологии и реанимации с операционным блоком ФГБУ “НМИЦ детской гематологии, онкологии и иммунологии им. Дмитрия Рогачева” Минздрава России. http://orcid.org/0000-0002-7945-2565. E-mail: schukinv@gmail.com

Жилкин Илья Владимирович - канд. мед. наук, врач-педиатр отделения онкологии и детской хирургии ФГБУ “НМИЦ детской гематологии, онкологии и иммунологии им. Дмитрия Рогачева” Минздрава России.

https://orcid.org/0000-0002-6922-1077. E-mail: izhilkin@gmail.com

Рабаев Гавриил Савельевич - врач-ординатор отделения онкологии и детской хирургии ФГБУ “НМИЦ детской гематологии, онкологии и иммунологии им. Дмитрия Рогачева” Минздрава России. https://orcid.org/0000-0002-56912522. E-mail: rabaevgesha@gmail.com

Моисеенко Роман Алексеевич - врач детский онколог отделения клинической онкологии ФГБУ “НМИЦ детской гематологии, онкологии и иммунологии им. Дмитрия Рогачева” Минздрава России. https://orcid.org/0000-0002-9654-084X. E-mail: doctor-moiseenko@yandex.ru

Грачев Николай Сергеевич - доктор мед. наук, заведующий отделением онкологии и детской хирургии ФГБУ “НМИЦ детской гематологии, онкологии и иммунологии им. Дмитрия Рогачева” Минздрава России.

https://orcid.org/0000-0002-4451-3233. E-mail: nick-grachev@yandex.ru

Для корреспонденции *: Ахаладзе Дмитрий Гурамович - 117997, Москва, ул. Саморы Машела, д. 1, Российская Федерация. Тел.: 8-905-587-89-92. E-mail: d.g.akhaladze@gmail.com 
Dmitry G. Akhaladze - Cand. of Sci. (Med.), Head of Thoracoabdominal Surgery Group, Dmitry Rogachev National Medical Research Center of Pediatric Hematology, Oncology and Immunology Ministry of Health of Russian Federation. https://orcid.org/0000-0002-1387-209X. E-mail: d.g.akhaladze@gmail.com

Denis Y. Kachanov - Doct. of Sci. (Med.), Head of the Department of Clinical Oncology, Dmitry Rogachev National Medical Research Center of Pediatric Hematology, Oncology and Immunology Ministry of Health of Russian Federation. https://orcid.org/0000-0002-3704-8783. E-mail: kachanov78@gmail.com

Natalya G. Uskova - Cand. of Sci. (Med.), Pediatric Surgeon of the Department of Pediatric Oncology and Surgery, Dmitry Rogachev National Medical Research Center of Pediatric Hematology, Oncology and Immunology Ministry of Health of Russian Federation. https://orcid.org/0000-0001-9424-1646. E-mail: nataliyauskova@gmail.com

Olga V. Melekhina - Cand. of Sci. (Med.), Surgeon, Department of Inteventional Radiology, Loginov Moscow Clinical Scientific Center, Department of Health of Moscow. https://orcid.org/0000-0002-3280-8667. E-mail: o.melekhina@mknc.ru

Nikolay N. Merkulov - Pediatric Surgeon of the Department of Pediatric Oncology and Surgery, Dmitry Rogachev National Medical Research Center of Pediatric Hematology, Oncology and Immunology Ministry of Health of Russian Federation. https://orcid.org/0000-0003-0404-6420. E-mail: dr.mernich@yandex.ru

Sergey R. Talypov - Cand. of Sci. (Med.), Pediatric Surgeon of the Department of Pediatric Oncology and Surgery, Dmitry Rogachev National Medical Research Center of Pediatric Hematology, Oncology and Immunology Ministry of Health of Russian Federation. http://orcid.org/0000-0002-5308-6544. E-mail: riumych@yandex.ru

Vladislav V. Schukin - Cand. of Sci. (Med.), Head of the Department of Anaesthesiology and Intensive Therapy with an Operating Unit, Dmitry Rogachev National Medical Research Center of Pediatric Hematology, Oncology and Immunology Ministry of Health of Russian Federation. http://orcid.org/0000-0002-7945-2565. E-mail: schukinv@gmail.com

Iliya V. Zhilkin - Cand. of Sci. (Med.), Pediatritian of the Department of Pediatric Oncology and Surgery, Dmitry Rogachev National Medical Research Center of Pediatric Hematology, Oncology and Immunology Ministry of Health of Russian Federation. https://orcid.org/0000-0002-6922-1077. E-mail: izhilkin@gmail.com

Gavriil S. Rabaev - Resident of the Department of Pediatric Oncology and Surgery, Dmitry Rogachev National Medical Research Center of Pediatric Hematology, Oncology and Immunology Ministry of Health of Russian Federation. https://orcid.org/0000-0002-5691-2522. E-mail: rabaevgesha@gmail.com

Roman A. Moiseenko - Pediatric Oncologist of the Department of Clinical Oncology, Dmitry Rogachev National Medical Research Center of Pediatric Hematology, Oncology and Immunology Ministry of Health of Russian Federation. https://orcid.org/0000-0002-9654-084X. E-mail: doctor-moiseenko@yandex.ru

Nikolay S. Grachev - Doct. of Sci. (Med.), Head of the Department of Pediatric Oncology and Surgery, Dmitry Rogachev National Medical Research Center of Pediatric Hematology, Oncology and Immunology Ministry of Health of Russian Federation. https://orcid.org/0000-0002-4451-3233. E-mail: nick-grachev@yandex.ru

For correspondence*: Dmitry G. Akhaladze - 1, Samory Mashela str., Moscow, 117997, Russian Federation. Phone: +7-905-587-89-92. E-mail: d.g.akhaladze@gmail.com

Статья поступила в редакцию журнала 15.06.2019. Принята к публикации 10.09.2019. Received 15 June 2019. Accepted for publication 10 September 2019. 